International Journal of Medical Sciences

ISSN 1449-1907 www.medsci.org 2007 4(1):1-6

Research Paper

(C) Ivyspring International Publisher. All rights reserved

\title{
Grb2-associated binder I polymorphism was associated with the risk of Helicobactor pylori infection and gastric atrophy
}

\author{
Yasuyuki Goto 1, Takafumi Ando 2, Kazuko Nishio 1, Sayo Kawai 1, Yoshiko Ishida 1, Mariko Naito 1, Hidemi \\ Goto 2 , Nobuyuki Hamajima 1
}

1. Department of Preventive Medicine / Biostatistics and Medical Decision Making, Nagoya University Graduate School of Medicine, Nagoya 466-8550, Japan

2. Department of Gastroenterology, Nagoya University Graduate School of Medicine, Nagoya 466-8550, Japan

Correspondence to: Yasuyuki Goto M.D., Ph.D., Department of Preventive Medicine / Biostatistics and Medical Decision Making, Nagoya University Graduate School of Medicine, 65 Tsurumai-cho, Showa-ku, Nagoya 466-8550 Japan. Tel: +81-52-744-2133, Fax: +81-52-744-2971, e-mail: y-goto@med.nagoya-u.ac.jp

Received: 2006.09.22; Accepted: 2006.10.25; Published: 2006.11.01

Background: Various single nucleotide polymorphisms (SNPs) have explained the association between Helicobacter pylori (H. pylori) and gastric atrophy and cancer. This study investigated the associations of Grb2 associated binder 1 (Gab1) polymorphism and the combination of PTPN11 gene encoding src homology 2 domain-containing protein tyrosine phosphatase-2 (SHP2) and Gab1 gene with gastric cancer and gastric atrophy among H. pylori seropositive subjects.

Methods: A single nucleotide polymorphism at intron 2 of Gab1 (JST164345) was examined for 454 Japanese health checkup examinees (126 males and 328 females) aged 35 to 85 without a history of gastric cancer and 202 gastric cancer patients (134 males and 68 females) aged 33 to 94 with pathologically confirmed diagnosis of gastric adenocarcinoma.

Results: The decreased OR of the Gab1 A/A for H. pylori seropositivity was 0.25 (95\% confidence interval (CI): $0.08-0.71$ ). Among seropositive healthy controls, the OR of the Gab1 G/A+A/A for gastric atrophy was significant (OR=1.95, 95\% CI: 1.12 -3.40). Seropositive individuals with PTPN11 G/G and Gab1 G/A+A/A demonstrated the highest risk of gastric atrophy with significance $(\mathrm{OR}=3.49,95 \% \mathrm{CI}$ : 1.54-7.90) relative to PTPN11 G/A+A/A and Gab1 G/G, the lowest risk combination, as a reference. However, the gene-gene interaction between PTPN11 and Gab1 was not observed (OR=1.39, 95\% CI: 0.41-4.66). Compared to gastric cancer case, the Gab1 did not influence the step of atrophy/metaplasia-gastric cancer sequence.

Conclusions: This study represents that the Gab1 polymorphism was associated with the low risk of H. pylori infection and the high risk of gastric atrophy among seropositive healthy controls, and that seropositive individuals with PTPN11 G/G and Gab1 G/A+G/G were associated with the greatest risk of gastric atrophy. These findings require confirmation in much larger studies.

Key words: Gab1, SHP-2, Polymorphism, Gastric atrophy, Helicobacter pylori infection

\section{Introduction}

Gastric cancer is the fourth most frequent cancer in the world, accounting for a large proportion of cancer cases in East Asia (China, Japan), Eastern Europe, and parts of Central and South America and it is the second most common cause of death from cancer [1]. Helicobacter pylori (H. pylori) strains carrying the cytotoxin-associated gene A (cagA) gene are strongly associated with increased risk of gastric adenocarcinoma [2]. However, only some of those infected developed H. pylori-related disease such as gastric ulcer, atrophy, cancer and so on. In Asian countries such as Japan with high prevalence of cagA-positive $H$. pylori infection, bacterial virulence factor has limitation of determining $H$. pylori-related disease. Therefore, we think it important to determine any host genetic predisposition to different outcome after the bacteria infection.
H. pylori, especially cagA positive strains, plays a crucial role in the development of gastric atrophy and cancer [2,3]. CagA/ src homology 2 domain-containing protein tyrosine phosphatase-2 (SHP-2) interaction elicits cellular changes that increase the risk of carcinogenesis via extracellular-regulated protein kinase (ERK) activity [4,5]. CagA is regarded as a bacterial protein that mimics mammalian docking/scaffolding molecule such as Grb2-associated binder 1 (Gab1) [6].

Gab1 consists of a pleckstrin homology domain, followed by a proline-rich region and multiple tyrosine phosphorylation sites that serve as binding sites for the SH2 domains of PI3-kinase, phospholipase $\mathrm{C}_{\gamma}, \mathrm{SHP} 2$, and CrkL $[7,8]$. Gab1 functions upstream of Ras/mitogen-activated proten kinase (MAPK) signaling pathway, most likely to regulate the activity of the GDP / GTP exchanger, in a variety of growth 
receptor signaling to activate ERK. SHP-2 is a ubiquitously expressed protein tyrosine phosphatase (PTPase) that contains two SH2 domains and an active catalytic domain $[9,10]$. SHP-2 PTPase activity is required for activation of the ERK subfamily of MAPK by epidermal growth factor (EGF) [11,12]. The previous reports suggest that the interaction between Gab1 and SHP2 is an essential component for ERK activation [13-17]. The activated SHP-2 is associated with Gab1 to mediate EGF-stimulated ERK2 activation, and Gab1 is the SHP-2 activator for the ERK MAP kinase pathway in EGF-stimulated cells [17].

We have reported previously that G/G of the PTPN11 gene encoding SHP-2 increased the risk of gastric atrophy among the seropositive subjects [18]. The present study examined the association of a polymorphism of Gab1 with gastric cancer and gastric atrophy identified as the precursor lesion of gastric cancer amongr the same Japanese subjects. There have been several SNPs identified in Gab1 gene in the Japan Single Nucleotide Polymorphisms (JSNP) database (http://snp.ims.u-tokyo.ac.jp). A particularly prevalent SNP is reported in intron 2 (G/A), identified as JST164345 in JSNP database. The gene-gene interaction between PTPN11 and Gab1 was also evaluated.

\section{Materials and methods}

\section{Patients}

The clinical characteristics of the subjects were described in our previous paper [18]. Briefly, the control group was 454 health checkup examinees (HCE) without a history of cancer (126 males and 328 females) aged 35 to 85 , who attended a health checkup program supported by the Nagoya municipal government, in August and September 2000. The study protocol was approved by the Ethics Committee of the Aichi Cancer Center, with which the chief investigation (N.H.) was affiliated at the enrollment of study subjects. The case group was 202 patients (134 males and 68 females) aged 33 to 94 with pathologically confirmed diagnosis of gastric adenocarcinoma, who underwent tumor resection in different affiliated hospitals of Nagoya University between January 1998 and June 2000. Informed consent was obtained from all the subjects. This study protocol was approved by the Ethics Committee of the Nagoya University Graduate School of Medicine.
Tests for Helicobacer pylori (H. pylori) antibody and pepsinogens

Anti-H. pylori IgG antibody tests, high-molecularweight campylobacter-associated-protein (HM-CAP) ELISA (Enteric Products Inc., Westbury, NY) and HM-CAP with antigens extracted from clinically isolated Japanese H. pylori strains (J-HM-CAP) ELISA (Kyowa Medex, Tokyo, Japan), were used for the identification of $H$. pylori-infected participants (in the control group the former was used and in the case group both were used.). An ELISA value of 2.3 or over was regarded as positive for both tests. The infection was confirmed in all gastric cancer cases by culture and bacteriological tests (Gram-negative, oxidase, catalase, and urease test-positive spiral, curved rods) using biopsy specimens before gastric resection. Pepsinogens I and II (PG I and PG II) in serum were measured by radioimmunoassay using a commercially available kit (DINABOT, Tokyo, Japan). Gastric atrophy was defined as PG $\mathrm{I}<70 \mathrm{ng} / \mathrm{ml}$ and PG I/PG II ratio <3.

\section{Genotype Assessment}

DNA was extracted from buffy coat fraction by Qiagen QIAamp DNA Blood Mini Kit (QIAGEN Inc.,Valencia,CA). A single nucleotide polymorphism (SNP) at intron 2 of Gab1, named as JST164345 in a database of Japan Single Nucleotide Polymorphisms (JSNP) at http://snp.ims.u-tokyo.ac.jp, was genotyped by PCR-CTPP (polymerase chain reaction with confronting two-pair primers) [19]. The primers were F1: 5' GGT TTA AAC TTT ATT CTG ACT GTT CCC, R1: 5' ACA CAA TTT AGT AAT AGC CAA AGT CAA C, F2: 5' GTT GTT GTG AAG TAG AAA CTG ATT TCT AA, and R2: 5' CTG GGG AGT GGG CCA. Genomic DNA was applied in a volume of $25 \mathrm{ul}$ with $0.12 \mathrm{mM}$ dNTPs, 25 pmol of each primer, 0.5 units of AmpliTaq Gold (Perkin-Elmer Corp., Foster City, CA), and 2.5 ul 10xPCR buffer including $15 \mathrm{mM}$ $\mathrm{MgCl} 2$. The PCR was performed with initial denaturation at $95{ }^{\circ} \mathrm{C}$ for 10 minutes, followed by 30 cycles of denaturation at $95{ }^{\circ} \mathrm{C}$ for 1 minute, annealing at $63.5{ }^{\circ} \mathrm{C}$ for 1 minute and extension at 72 ${ }^{\circ} \mathrm{C}$ for 5 minutes. The final extension was at $72{ }^{\circ} \mathrm{C}$ for 5 minutes. Figure 1 shows the results of PCR-CTPP for Gab1. PCR product was visualized on a $2 \%$ agarose gel with ethidium bromide staining. A SNP of PTPN11 that encodes SHP-2 (JST057927) was genotyped as reported in our previous report [19].

Figure $12 \%$ agarose gel showing the different genotypes for the Gabl polymorphism.

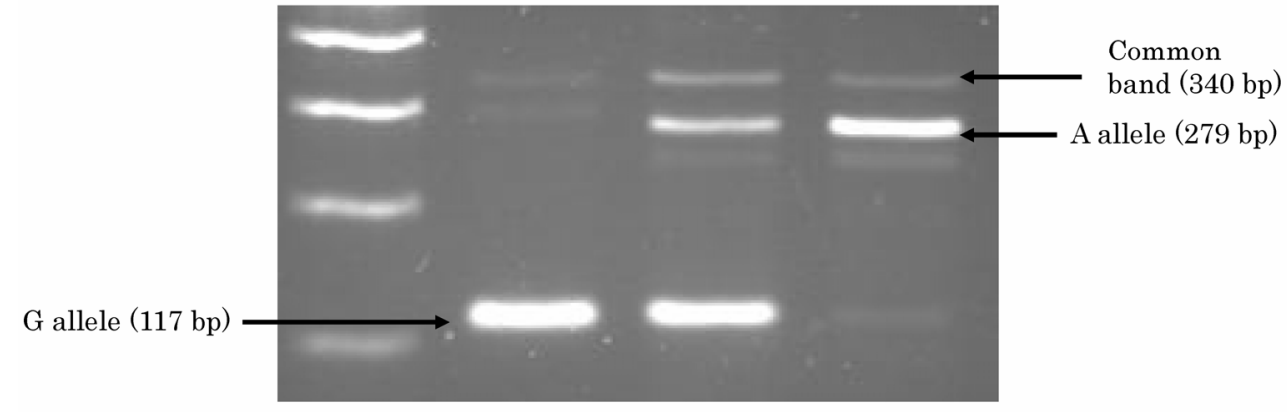




\section{Statistical analysis}

To prevent confounding bias, odds ratios (ORs) adjusted for sex and age with 95\% confidence intervals (CIs) were calculated using logistic regression analysis.

The Hardy- Weinberg equilibrium was examined for discrepancy between genotype and allele distributions using a $\chi^{2}$ test.

The product variable between gene and gene was included in the logistic model to evaluate the multiplicative interactive effect of genes. All tests were 2-tailed with statistical significance setting at the level of $p<0.05$. Hardy-Weinberg equilibrium was tested for the Gab1 polymorphism. These calculations were performed by computer program STATA Version 8 (STATA Corp, College Station, TX).

\section{Results}

\section{Study characteristics}

Although these summary has been described previously [18], it is shown here again for the readers' convenience (Table 1). The prevalence of $H$. pylori seropositivity was significantly higher in the gastric cancer cases than in the healthy controls $(100 \%$ vs. $55.1 \%, \mathrm{p}<0.001)$. One hundred seventy-nine of the 202 $(89 \%)$ gastric cancer cases had atrophy with significantly higher prevalence than the control group (35\%). Atrophy was present in $54.8 \%$ of the $250 \mathrm{H}$. pylori seropositive healthy controls, which was significantly lower than the seropositive gastric cancer cases $(p=0.001)$ but significantly higher than the seronegative healthy controls in which atrophy was present in only $10 \%$ of subjects $(\mathrm{p}<0.001)$.

Table 1 Characteristics of the study subjects by source of recruitment.

\begin{tabular}{|c|c|c|}
\hline & $\begin{array}{l}\text { Cases } \\
\text { n(\%) }\end{array}$ & $\begin{array}{c}\text { Controls } \\
\mathbf{n}(\%)\end{array}$ \\
\hline Number of subjects & 202 & 454 \\
\hline $\begin{array}{c}\text { Age in years } \\
\text { (mean } \pm \text { standard } \\
\text { deviation) }\end{array}$ & $66.7 \pm 12.3$ & $58.4 \pm 11.9$ \\
\hline \multicolumn{3}{|l|}{ Sex } \\
\hline Male & $134(66.3)$ & $126(27.8)$ \\
\hline Female & $68(33.7)$ & $328(72.3)$ \\
\hline \multicolumn{3}{|l|}{ H. pylori antibody } \\
\hline Negative & $0(0)$ & $204(44.9)$ \\
\hline Positive & $202(100)$ & $250(55.1)$ \\
\hline \multicolumn{3}{|l|}{ Gastric atrophy } \\
\hline Negative & $23(11.4)$ & $296(65.2)$ \\
\hline Positive & $179(88.6)$ & $158(34.8)$ \\
\hline
\end{tabular}

\section{Gab1 polymophism and H. pylori infection risk}

The Gab1 genotype distribution of the control group was in the Hardy-Weinberg equilibrium $\left(\mathrm{X}^{2}=2.50, \mathrm{P}=0.11\right)$. Table 2 shows that the genotype frequency and odds ratio (OR) of $H$. pylori seropositivity in healthy controls. The seropositivity rate for those with A/A was lowest. The decreased OR of A/A for H. pylori seropositivity was 0.25 (95\% CI: 0.08-0.71). Twenty-one of the 204 seronegative healthy controls $(10 \%)$ had atrophy, which were considered as the loss of $H$. pylori infection following sever atrophy. To ascertain whether the reduced infection risk related to this polymorphism follows sever atrophy, we arranged category so as to classify 21 seronegative controls with atrophy as seropositive controls. We calculated the OR for H. pylori again and the corresponding OR was also a decreased risk with significance (OR=0.25, 95\% CI: 0.09-0.71).

Table 2 Genotype frequency and odds ratios (ORs) and 95\% confidence intervals (95\%CIs) of $H$. pylori Seropositivity $(\mathrm{HP}+)$ in Healthy Checkup Examinees.

\begin{tabular}{|c|c|c|c|}
\hline Genotype & $\mathbf{n}$ & $\mathbf{H P +} \mathbf{( \% )}$ & $\mathbf{O R} \mathbf{a}(\mathbf{9 5} \% \mathbf{C I})$ \\
\hline G/G & 317 & $171(53.9)$ & 1.00 (Reference) \\
G/A & 119 & $74(62.2)$ & $1.30(0.81-2.07)$ \\
\hline A/A & 18 & $5(27.8)$ & $0.25(0.08-0.71)$ \\
\hline G/A+A/A & 137 & $79(57.7)$ & $1.02(0.67-1.56)$ \\
\hline
\end{tabular}

aSex-age-adjusted odds ratio

The combination of Gabl and PTPN1 land $\mathrm{H}$. pylori-related gastric atrophy

We have got subjects narrowed down to $H$. pylori seropositive healthy controls because of our interest in the association between these polymorphisms and digestive disease caused by $H$. pylori infection. We have reported previously that $G / G$ of the PTPN11 gene encoding SHP-2 increased the risk of gastric atrophy [18]. Table 3 shows the age-sex-adjusted ORs of the Gab1 and the combinations of PTPN11 and Gab1 genotypes for gastric atrophy among seropositive healthy controls. The OR of $\mathrm{G} / \mathrm{A}+\mathrm{A} / \mathrm{A}$ for gastric atrophy was significant $(\mathrm{OR}=1.95,95 \% \mathrm{CI} 1.12-3.40)$. Seropositive individuals with the PTPN11 G/G and Gab1 G/A+A/A demonstrated the highest risk of gastric atrophy with significance relative to PTPN11 $\mathrm{G} / \mathrm{A}+\mathrm{A} / \mathrm{A}$ and $\mathrm{Gab1} \mathrm{G} / \mathrm{G}$, the lowest risk combination, as a reference. However, the gene-gene interaction between the PTPN11and the Gab1 was not observed (OR=1.39, 95\% CI 0.41-4.66).

Table 3 ORs and 95\% CIs for gastric atrophy (GA) of Gabl and the combinations of PTPN11 and Gabl genotypes among seropositive healthy controls.

\begin{tabular}{|c|c|c|c|c|}
\hline \multicolumn{2}{|c|}{ Genotype } & $n$ & GA (\%) & $\mathrm{OR}^{\mathrm{a}}(\mathbf{9 5} \% \mathrm{CI})$ \\
\hline \multicolumn{5}{|c|}{ Gab1 } \\
\hline \multicolumn{2}{|c|}{$\mathrm{G} / \mathrm{G}$} & 171 & 85 (49.7) & 1.00 (Reference) \\
\hline \multicolumn{2}{|c|}{$\mathrm{G} / \mathrm{A}$} & 74 & $48(64.9)$ & $1.87(1.06-3.29)$ \\
\hline \multicolumn{2}{|c|}{$\mathrm{A} / \mathrm{A}$} & 5 & $4(80.0)$ & $4.24(0.45-39.7)$ \\
\hline \multicolumn{2}{|c|}{$\mathrm{G} / \mathrm{A}+\mathrm{A} / \mathrm{A}$} & 79 & $52(65.8)$ & $1.95(1.12-3.40)$ \\
\hline \multicolumn{2}{|c|}{ Total } & 250 & $137(54.8)$ & \\
\hline PTPN11 ${ }^{b}$ & Gab1 & & & \\
\hline $\mathrm{G} / \mathrm{A}+\mathrm{A} / \mathrm{A}$ & $\mathrm{G} / \mathrm{G}$ & 49 & $20(40.8)$ & 1.00 (Reference) \\
\hline $\mathrm{G} / \mathrm{A}+\mathrm{A} / \mathrm{A}$ & $\mathrm{G} / \mathrm{A}+\mathrm{A} / \mathrm{A}$ & 23 & $12(52.2)$ & $1.57(0.58-4.29)$ \\
\hline $\mathrm{G} / \mathrm{G}$ & $\mathrm{G} / \mathrm{G}$ & 121 & $64(52.9)$ & $1.60(0.82-3.15)$ \\
\hline $\mathrm{G} / \mathrm{G}$ & $\mathrm{G} / \mathrm{A}+\mathrm{A} / \mathrm{A}$ & 55 & $39(70.9)$ & $3.49(1.54-7.90)$ \\
\hline \multicolumn{2}{|c|}{ Total } & 248 & $135(54.4)$ & \\
\hline
\end{tabular}

aSex-age-adjusted odds ratio

'Two subjects could not be genotyped for PTPN11

Gab1 polymorphism and the step of

atrophy/metaplasia-gastric cancer

We assessed whether the Gab1 gene polymorphism was associated with the development of H. pylori-related gastric cancer. Table 4 shows the 
genotype frequency and ORs of the Gab1 genotypes for gastric cancer among the seropositive subjects. The Gab1 gene was not associated with the risk of gastric cancer among the seropositive subjects. In order to find out if the Gab1 polymorphism influenced the step of atrophy/metaplasia-gastric cancer sequence, proposed as the Correa cascade [20], we made a comparison between cases and controls with seropositive atrophy. The ORs of the G/A and A/A for gastric cancer was 1.05 (95\% CI 0.63-1.74) and 1.05 (95\% CI 0.27-4.07), respectively.

Table 4 The Gabl genotype frequency and ORs for gastric cancer among the seropositive subjects.

\begin{tabular}{|c|c|c|c|}
\hline Genotype & $\begin{array}{c}\text { Cases } \\
\mathbf{n}\end{array}$ & $\begin{array}{c}\text { Controls } \\
\mathbf{n}\end{array}$ & OR $^{\mathbf{a}} \mathbf{( 9 5 \% \mathbf { C I } )}$ \\
\hline $\mathrm{G} / \mathrm{G}$ & 119 & 171 & 1.00 (Reference) \\
\hline G/A & 75 & 74 & $1.35(0.88-2.07)$ \\
\hline A/A & 8 & 5 & $1.53(0.46-5.10)$ \\
\hline $\begin{array}{c}\text { G/A+A/A } \\
\text { Total }\end{array}$ & 83 & 79 & $1.36(0.90-2.06)$ \\
\hline
\end{tabular}

aSex-age-adjusted odds ratio

\section{Discussion}

This epidemiologic finding that the Gab1 polymorphism was associated with $H$. pylori seropositivity and gastric atrophy is plausible. However, the Gab1 polymorphism did not influence the development of gastric cancer.

The Gab1 A/A decreased the risk of H. pylori seropositivity, whereas the Gab1 G/A+A/A was associated with gastric atrophy risk. In order to find out if the Gab1 polymorphism relates to loss of $H$. pylori infection following severe atrophy, which has been reported [21], we classified 21 seronegative controls with atrophy, which might be eradicated naturally, as seropositive controls. If the decreased OR for $H$. pylori seropositivity under this condition is not significant, we could conclude that the Gab1 polymorphism is associated with severe atrophy which induces the chance of natural eradication of the bacteria. However, the OR of the A/A genotype for $H$. pylori seropositivity was 0.25 (95\% CI 0.09-0.71). So, the Gab1 polymorphism was associated with the low risk of the infection independent on severe atrophy. Although the Gab1 polymorphism prevented H. pylori infection, it was associated with the risk of atrophy identified as the precursor lesion of gastric cancer but not severe atrophy which is a final stage of atrophic status and loses H. pylori infection. Gab1 plays important roles in the signal transduction of cytokines, growth factors, antigen receptors [22]. The association between the polymorphism of cytokine genes such as interleukin $1 B[23,24]$ and tumor necrosis factor $A[25,26]$ and $H$. pylori seropositivity have been reported. Gab1 may affect the $H$. pylori infection through the level of cytokines which are advantageous to eradication of $H$. pylori.

These results should be interpreted with caution. The question is whether these apparently $H$. pylori-negative subjects were indeed truly negative or they eventually led to loss of the infection due to sever gastric atrophy. Because, in our study, gastric atrophy and $H$. pylori infection were based on serological diagnosis and the diagnosis happened to be imperfect, we might not discriminate severe atrophy exactly and further biological studies with histological assessment are needed to confirm the association. The functional changes caused by Gab1 polymorphism are not known and may be linkage disequilibrium with another gene. These are deficiencies of this study but we hope that our epidemiological and biologically plausible observation would stimulate interest in the study of the molecular mechanisms of action of this polymorphism. Because these results were based on the low frequency of $A / A$, they might be inconsistent due to the random errors. Studies of a larger size are needed to confirm our finding. This study, however, had $80 \%$ power to detect an absolute difference in the frequency of $\mathrm{G} / \mathrm{A}+\mathrm{A} / \mathrm{A}$, given $44 \%$ in the case and $56 \%$ in the control.

The PTPN11 polymorphism was associated with gastric atrophy among seropositive subjects [18]. The two tyrosine residues (Tyr-627 and Tyr-659) in the carboxyl-terminal region of Gab1 are required for SHP-2 binding to Gab1 and for EGF-stimulated ERK activation, in which another study reported Gab1/SHP-2 interaction was independent of ERK activation [27]. Tyr-627 and Tyr-659 of Gab1 constitute a bisphosphoryl tyrosine-based activation motif (BTAM) that binds to and activates SHP-2 [17]. Two SH2 domaines of SHP-2, termed N-SH2 and C-SH2 domaines, are arranged in tandem at the amino (N)-terminal portion. SHP-2 has a low basal PTPase activity that can be activated by deletion of N-SH2 or both SH2 domains or by specific phosphopeptides that bind to the $\mathrm{SH} 2$ domains. The tandem $\mathrm{SH} 2$ domains bind to Tyr-627 and Tyr-659 simultaneously in a specific orientation, in which Tyr-627 binds to the $\mathrm{N}-\mathrm{SH} 2$ domain and Tyr-659 binds to the C-SH2 domain [17]. Experiments with Gab1 mutants which are unable to bind to SHP-2 indicate that the interaction between SHP-2 and Gab1 and the activation of SHP-2 are essential for ERK activation [28-30]. The Gab1 polymorphism may affect the interaction with SHP-2 through the mechanism such as BTAM after $H$ pylori infection, resulting in influencing the abnormal proliferation and movement of gastric epithelial cells related to gastric atrophy via the activation of ERK.

This study also showed that the gastric atrophy risk was highest for those who carry the PTPN11 G/G and Gab1 G/A+A/A among seropositive subjects without interaction between those genotypes. Considering the mechanism such as BTAM, the epidemiologic interaction was expected, but not observed.

Among the seropositive subjects, the Gab1 polymorphism was not associated with gastric cancer. When we made a comparison between cases and controls with seropositive atrophy, the ORs of G/A and A/A for gastric cancer were also each insignificant. This result showed that this 
polymorphism did not influence the step of atrophy/metaplasia-gastric cancer sequence. As discussed in our previous report [18], our all gastric cancer cases had evidence of $H$. pylori infection because of the earlier presentation or diagnosis of cancer.

In summary, the Gab1 A/A was associated with the low risk of $H$. pylori infection while the G/A and A/A genotypes together may increase the risk for gastric atrophy. The biological mechanism of this polymorphism remains to be elucidated. In addition, individuals with the PTPN11 G/G and Gab1 $\mathrm{G} / \mathrm{A}+\mathrm{A} / \mathrm{A}$ demonstrated the greatest risk of gastric atrophy with no interaction. Our data provided further evidence for host genetic factors in the susceptibility to $H$ pylori infection and $H$. pylori-related gastric atrophy. Further investigation of the association requires much larger studies, as well as confirmatory biological studies with histological assessment.

\section{Acknowledgments}

The authors are grateful to Dr. Nobuyuki Katsuda for the enrollments of controls, and Ms. Yoko Mitsuda and Ms. Mayumi Kato for their technical assistance. This work was supported in part by a Grant-in-Aid for Scientific Research on Special Priority Areas of Cancer from the Ministry of Education, Culture, Sports, Science and Technology of Japan.

\section{Conflicts of interest}

The authors have declared that no conflict of interest exists.

\section{References}

1. Parkin DM, Bray F, Ferlay J, Pisani P. Global cancer statistics, 2002. CA Cancer J Clin 2005; 55:74-108.

2. Blaser MJ, Perez-Perez GI, Kleanthous H, et al. Infection with Helicobacter pylori strains possessing cagA is associated with an increased risk of developing adenocarcinoma of the stomach. Cancer Res 1995; 55: 2111-5.

3. Uemura N, Okamoto S, Yamamoto S, et al. Helicobacter pylori infection and the development of gastric cancer. N Engl J Med 2001; 345: 784-9.

4. Higashi H, Tsutsumi $\mathrm{R}$, Muto $\mathrm{S}$, et al. SHP-2 tyrosine phosphatase as an intracellular target of Helicobacter pylori CagA protein. Science 2002; 295: 683-6.

5. Higashi H, Nakaya A, Tsutsumi R, et al. Helicobacter pylori CagA induces Ras-independent morphogenetic response through SHP-2 recruitment and activation. J Biol Chem 2004; 279: 17205-16.

6. Hatakeyama M. Helicobacter pylori CagA--a potential bacterial oncoprotein that functionally mimics the mammalian Gab family of adaptor proteins. Microbes Infect. 2003; 5: 143-150.

7. Hibi M, Hirano T. Gab-family adapter molecules in signal transduction of cytokine and growth factor receptors, and $\mathrm{T}$ and B cell antigen receptors. Leuk Lymphoma 2000; 37: 299-307.

8. Liu Y, Rohrschneider LR. The gift of Gab1. FEBS lett 2002; 515: 1-7.

9. Feng GS, Hui CC, Pawson T. SH2-containing phosphotyrosine phosphatase as a target of protein-tyrosine kinases. Science 1993; 259: 1607-11.

10. Vogel W, Lammers R, Huang J, Ullrich A. Activation of a phosphotyrosine phosphatase by tyrosine phosphorylation. Science 1993; 259: 1611-4.
11. Deb TB, Wong L, Salomon DS, et al. A common requirement for the catalytic activity and both $\mathrm{SH} 2$ domains of SHP-2 in mitogen-activated protein (MAP) kinase activation by the ErbB family of receptors. a specific role for SHP-2 in MAP, but not c-Jun amino-terminal kinase activation. J Biol Chem 1998; 273: 16643-6.

12. Bennett AM, Hausdorff SF, O'Reilly AM, et al. Multiple requirements for SHPTP2 in epidermal growth factor-mediated cell cycle progression. Mol Cell Biol 1996; 16: 1189-202.

13. Cunnick JM, Dorsey JF, Munoz-Antonia T, et al. Requirement of SHP2 binding to Grb2-associated binder-1 for mitogen-activated protein kinase activation in response to lysophosphatidic acid and epidermal growth factor. J Biol Chem 2000; 275: 13842 - 8.

14. Ymazaki S, Nishida K, Yoshida $Y$, et al. Gab1 is required for EGF receptor signaling and the transformation by activated ErbB2. Oncogene 2003; 22: 1546-56.

15. Schaeper U, Gehring NH, Fuchs KP, et al. Coupling of Gab1 to c-Met, Grb2, and Shp2 mediates biological responses. J Cell Biol 2000; 149: 1419-32.

16. Maroun CR, Naujokas MA, Holgado-Madruga M, et al. The tyrosine phosphatase SHP-2 is required for sustained activation of extracellular signal-regulated kinase. Mol Cell Biol 2000; 20: 8513-25.

17. Cunnick JM, Mei L, Doupnik CA, Wu J. Phosphotyrosines 627 and 659 of Gab1 constitute a bisphosphoryl tyrosine-based activation motif (BTAM) conferring binding and activation of SHP2. J Biol Chem 2001; 276: 24380-7.

18. Goto Y, Ando T, Yamamoto K, et al. Association between the precursor lesion of gastric cancer and polymorphism of PTPN11 encoding SHP-2 among Helicobacter pylori seropositive Japanese. Int J Cancer. 2006; 118: 203-208.

19. Hamajima N, Saito $T$, Matsuo $K$, et al. Polymerase chain reaction with confronting two-pair primers for polymorphism genotyping. Jpn J Cancer Res 2000; 91: 865-68.

20. Correa P, Haenszel W, Cuello C, et al. A model for gastric cancer epidemiology. Lancet 1975; 2: 58-9.

21. Asaka M, Sugiyama T, Nobuta A, et al. Atrophic gastritis and intestinal metaplasia in Japan: results of a large multicenter study. Helicobacter 2001; 6: 294-9.

22. Nishida K, Yoshida $Y$, Itoh $\mathrm{M}$, et al. Gab-family adapter proteins act downstream of cytokine and growth factor receptors and T- and B-cell antigen receptors. Blood. 1999; 93: 1809-1816.

23. Hamajima $\mathrm{N}$, Matsuo $\mathrm{K}$, Saito $\mathrm{T}$, et al. Interleukin 1 polymorphisms, lifestyle factors, and Helicobacter pylori infection. Jpn J Cancer Res. 2001; 92: 383-9.

24. Uno M, Hamajima N, Ito LS, et al. Helicobacter pylori seropositivity and IL-1B C-31T polymorphism among Japanese Brazilians. Int J Mol Med. 2002; 10: 321-326.

25. Yea SS, Yang YI, Jang WH, et al. Association between TNF-alpha promoter polymorphism and Helicobacter pylori cagA subtype infection. J Clin Pathol. 2001; 54: 703-706.

26. Hamajima N, Shibata A, Katsuda N, et al. Subjects with TNF-A-857TT and -1031TT genotypes showed the highest Helicobacter pylori seropositive rate compared with those with other genotypes. Gastric Cancer. 2003; 6: 230-236.

27. Holgado-Madruga M, Wong AJ. Role of the Grb2-associated binder 1/SHP-2 interaction in cell growth and transformation. Cancer Res 2004; 64: 2007-15.

28. Schaeper U, Gehring NH, Fuchs KP, et al. Coupling of Gab1 to c-Met, Grb2, and Shp2 mediates biological responses. J Cell Biol. 2000; 149: 1419-1432.

29. Maroun CR, Naujokas MA, Park M. Membrane targeting of Grb2-associated binder-1 (Gab1) scaffolding protein through Src myristoylation sequence substitutes for Gab1 pleckstrin homology domain and switches an epidermal growth factor 
response to an invasive morphogenic program. Mol Biol Cell. 2003; 14: 1691-1708.

30. Cunnick JM, Dorsey JF, Munoz-Antonia T, et al. Requirement of SHP2 binding to Grb2-associated binder-1 for mitogen-activated protein kinase activation in response to lysophosphatidic acid and epidermal growth factor. J Biol Chem. 2000; 275: 13842-8. 